\title{
Impact of rituximab on COVID-19 outcomes
}

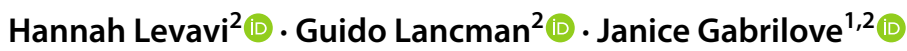

Received: 20 April 2021 / Accepted: 6 September 2021 / Published online: 22 September 2021

(c) The Author(s), under exclusive licence to Springer-Verlag GmbH Germany, part of Springer Nature 2021

\begin{abstract}
Rituximab is associated with prolonged B-cell depletion and secondary hypogammaglobulinemia and is associated with a dampened humoral response and increased infectious complications. To describe the potential impact of prior rituximab therapy on clinical outcomes from SARS-CoV-2 infection and development of COVID-19 antibodies, we conducted a retrospective study of adults across the Mount Sinai Health System diagnosed with COVID-19 who received rituximab for any indication from February 2019 to October 2020. Patients' baseline characteristics, markers of disease severity, clinical outcomes, and antibody development were examined. Of the 49 patients included in the analysis, $63.2 \%$ required hospitalization for COVID-19, 24.5\% required an ICU admission, and 32.7\% died. Proximity of last rituximab infusion and COVID19 diagnosis did not affect rates of hospitalization, admission to intensive care units or death. Over half (51.7\%) of those whose antibodies were checked developed neutralizing anti-spike protein antibodies. The median time between rituximab administration and COVID-19 diagnosis was not significantly different between those who developed antibodies and those who did not $(p=.323)$. Of the 14 patients with documented negative COVID-19 antibody titers, 11 of them survived SARSCoV-2 infection, indicating that development of neutralizing antibodies may not be necessary for recovery from COVID-19.
\end{abstract}

Keywords COVID-19 $\cdot$ SARS-CoV-2 $\cdot$ Rituximab $\cdot$ Humoral immunity $\cdot$ T-cell immunity $\cdot$ B-lymphocytes

\section{Introduction}

Since the emergence of the COVID-19 pandemic, much has been learned about the complex immune response to SARS-CoV-2 infection [1]. Successful viral clearance is linked to careful coordination between antibody-producing B-cells, CD4 + T-cells, and CD8 + T-cells, while asynchrony among these branches of the adaptive immune system has been implicated in poor clinical outcomes [2].

Rituximab is a chimeric monoclonal antibody that targets the CD-20 antigen on B-lymphocytes and is indicated for the treatment of a number of hematologic and nonhematologic conditions. The administration of rituximab is associated with rapid B-cell depletion and secondary hypogammaglobulinemia, recovery from which may take up to 12 months [3]. During this period of lymphopenia,

Hannah Levavi

hannah.levavi@mountsinai.org

1 Tisch Cancer Institute, New York, NY, USA

2 Division of Hematology and Medical Oncology, Icahn School of Medicine, One Gustave L. Levy Place, Box 1079, New York, NY 10029-6574, USA patients are susceptible to a well-described increase in infectious complications due to impaired opsonization and an inability to generate antibodies in response to new antigens [4]. Given rituximab's long-lasting effects on the humoral response to infection, we sought to evaluate the impact of prior rituximab therapy on antibody formation to COVID-19 and clinical outcomes from SARS-CoV-2 infection.

\section{Methods}

We conducted a retrospective study of adult patients diagnosed with COVID-19 and who had received rituximab for any indication from February 2019 to October 2020, across the Mount Sinai Health System (MSHS). A confirmed case of COVID-19 was defined as a positive result on a reverse transcriptase polymerase chain reaction (RT-PCR) SARSCoV-2 assay obtained by nasopharyngeal swab using the Roche cobas $6800 \operatorname{System}(n=55)$ [5], Panther Fusion $(n=1)$ [6], Cepheid GeneXpert (Cedano) $(n=1)$ [7], or Simplexa $(n=1)$ [8]. Patients were included in the analysis regardless of the presence or absence of COVID-19-related symptoms at the time of RT-PCR testing, but only one patient (1/58) 
was asymptomatic at the time of testing. Patients who were hospitalized or had been seen in the emergency department, or ambulatory setting, were included in the analysis. Patients were identified using a data mining tool within the electronic medical record capable of extracting records of patients who met both the criteria of having a recorded rituximab administration in the selected time period and a positive RT-PCR test for SARS-CoV-2. A comprehensive chart review was conducted to evaluate baseline characteristics, assess infection severity, identify disease outcomes, and delineate antibody development.

The antibody assay used at our institution is an ELISA assay that detects SARS-CoV-2 IgG spike antibodies. Reported sensitivity is $92.5 \%$, and specificity is $100 \%$, with a positive predictive value (PPV) of $100 \%$ and a negative predictive value (NPV) of $99.6 \%$ [9]. Thirteen patients were tested for the presence of COVID-19 antibodies more than once. In those patients who had multiple tests in which COVID-19 antibodies were detected $(n=3)$, the date of the earliest positive test was recorded. In those patients who had multiple tests in which COVID-19 antibodies were not detected $(n=6)$, the date of the test farthest from COVID19 diagnosis was recorded. In patients who tested positive and later tested negative for the presence of COVID-19 antibodies $(n=2)$, only the date of the earliest positive test was recorded. In those patients who tested negative and later tested positive for the presence of COVID-19 antibodies $(n=2)$, only the date of the earliest positive test was recorded. Statistical analysis was performed using chi-square analyses, Mood's median tests, Fisher exact tests, and calculating relative risks [10].

The study was approved by the Program for the Protection of Human Subjects at the Icahn School of Medicine at Mount Sinai.

\section{Results}

Fifty-eight patients diagnosed with COVID-19 who had received rituximab in the specified time period were identified. Nine patients were excluded from the analysis: two patients were excluded because they were under age 18 at the time of COVID-19 diagnosis, 6 patients were removed from the analysis because rituximab was administered after COVID-19 diagnosis, and 1 patient was excluded because dates for rituximab administration as well as other information was not available in the electronic medical record (Fig. 1).

Baseline patient characteristics are provided in Table 1. Twenty-one (42.9\%) patients received rituximab for malignant disease, while 28 patients $(57.1 \%)$ received the medication for autoimmune or rheumatic disorders. Thirty-four

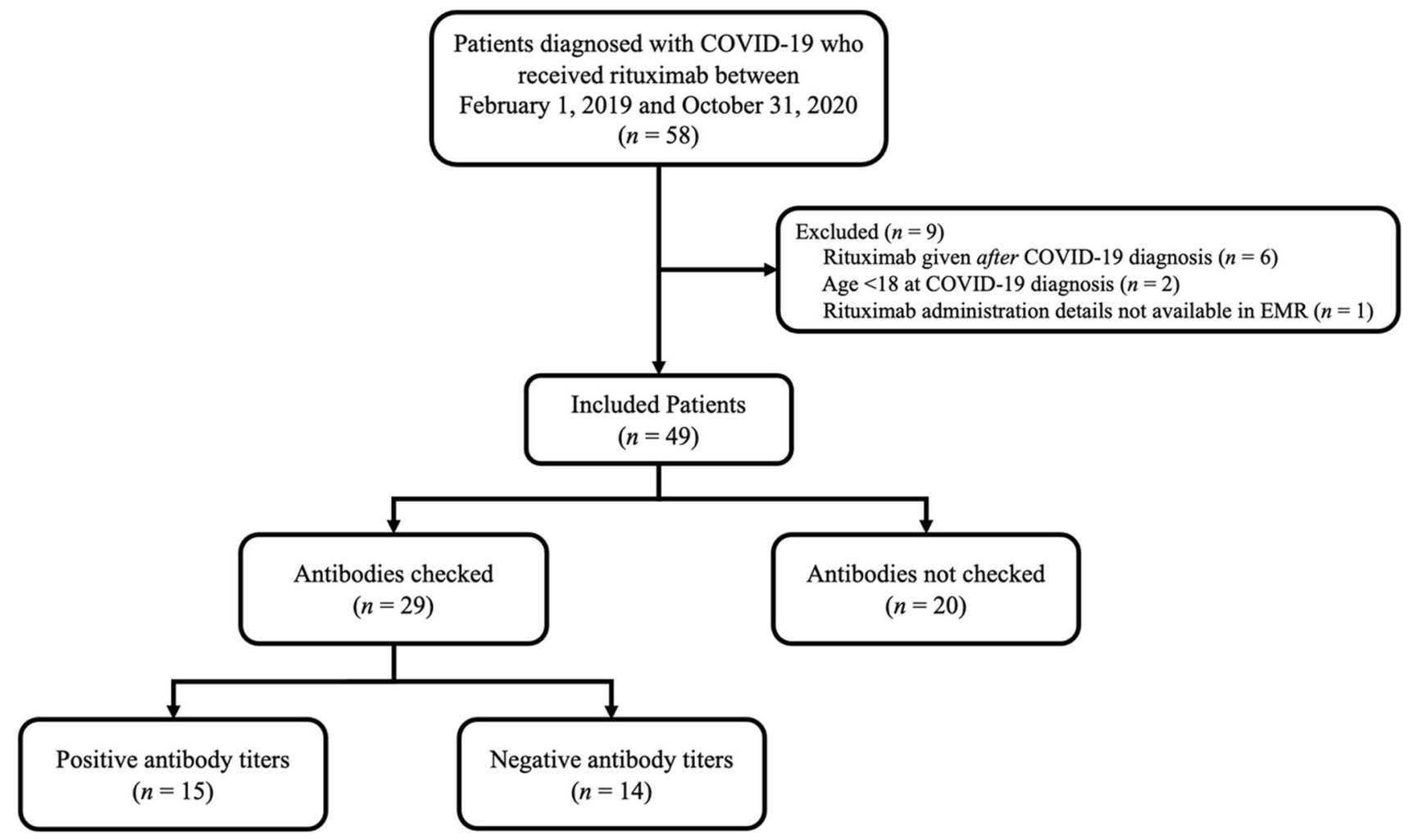

Fig. 1 Consort diagram 
Table 1 Baseline characteristics

\begin{tabular}{|c|c|}
\hline Characteristic & All patients $(n=49)$ \\
\hline Median age at COVID-19 diagnosis, years (range) & $56(30-85)$ \\
\hline Female sex, $n(\%)$ & $25(51.0)$ \\
\hline \multicolumn{2}{|l|}{ Race, $n(\%)$} \\
\hline White & $17(34.7)$ \\
\hline Other & $17(34.7)$ \\
\hline African American & $10(20.4)$ \\
\hline Unknown & $5(10.2)$ \\
\hline \multicolumn{2}{|l|}{ Ethnicity, $n(\%)$} \\
\hline Non-Hispanic & $27(55.1)$ \\
\hline Hispanic & $17(34.7)$ \\
\hline Unknown & $5(10.2)$ \\
\hline \multicolumn{2}{|l|}{ Baseline comorbidities, $n(\%)$} \\
\hline Diabetes & $15(30.6)$ \\
\hline Hypertension & $19(38.8)$ \\
\hline Obesity (BMI $\geq 35)$ & $10(20.4)$ \\
\hline Hyperlipidemia & $26(53.1)$ \\
\hline History of ASCVD & $8(16.3)$ \\
\hline \multicolumn{2}{|l|}{ Rituximab indication, $n(\%)$} \\
\hline Non-Hodgkin's lymphoma & $18(36.7)$ \\
\hline Hodgkin's lymphoma & $1(2.0)$ \\
\hline CD20 + B-cell ALL & $2(4.1)$ \\
\hline Autoimmune hematologic disorders (ITP, AIHA, pure red cell aplasia) & $5(10.2)$ \\
\hline Rheumatoid Arthritis & $5(10.2)$ \\
\hline $\begin{array}{l}\text { Autoimmune neurologic diseases (myasthenia gravis, autoimmune encephalitis, } \\
\text { CIDP, MS, NMOSD) }\end{array}$ & $5(10.2)$ \\
\hline Vasculitis & $4(8.2)$ \\
\hline Inflammatory myopathy & $3(6.1)$ \\
\hline Bullous skin disorders & $2(4.1)$ \\
\hline Antibody-mediated solid organ transplant rejection & $2(4.1)$ \\
\hline Lupus & $1(2.0)$ \\
\hline Sarcoidosis & $1(2.0)$ \\
\hline Median number of rituximab doses since 2/2019 (range) & $4(1-10)$ \\
\hline \multicolumn{2}{|l|}{ COVID-19-directed treatments, $n(\%)$} \\
\hline Hydroxychloroquine & $28(57.1)$ \\
\hline Azithromycin & $26(53.1)$ \\
\hline Steroids & $15(30.6)$ \\
\hline Remdesivir & $10(20.4)$ \\
\hline Tocilizumab & $1(2.0)$ \\
\hline Convalescent plasma & $6(12.2)$ \\
\hline
\end{tabular}

$B M I$ body mass index, $A S C V D$ atherosclerotic cardiovascular disease, $B$ - $A L L$ B-cell acute lymphoblastic leukemia/lymphoma, ITP immune thrombocytopenic purpura, AIHA autoimmune hemolytic anemia, CIDP chronic inflammatory demyelinating polyneuropathy, $M S$ multiple sclerosis, NMOSD neuromyelitis optica spectrum disorder

(69.4\%) patients received other concurrent immunodepleting or immunomodulatory therapies at the time of their rituximab treatments, including chemotherapy, monoclonal antibodies, and tyrosine kinase inhibitors (Table 2 ).

More than half $(57.1 \%, n=28)$ of the patients in this analysis had received their last rituximab dose less than
3 months prior to COVID-19 diagnosis; 13 (26.5\%) patients had received rituximab 3-6 months prior to COVID-19 diagnosis, and the remaining 8 (16.3\%) patients received rituximab $>6$ months prior to contracting SARS-CoV-2 (Table 3). 
Table 2 Chemotherapy, immunosuppressants, and immunomodulators given concurrently with rituximab

\begin{tabular}{ll}
\hline Agent & $n^{\mathrm{a}}$ \\
\hline Anti-thymocyte globulin & 1 \\
Azathioprine & 1 \\
Belatacept & 1 \\
Bendamustine & 1 \\
Cyclophosphamide & 13 \\
Cyclosporine & 1 \\
Doxorubicin & 13 \\
Etoposide & 4 \\
Leflunomide & 1 \\
Lenalidomide & 1 \\
Mesalamine & 1 \\
Methotrexate & 8 \\
Mycophenolate mofetil & 4 \\
Steroids & 22 \\
Tacrolimus & 4 \\
Vincristine & 15 \\
\hline
\end{tabular}

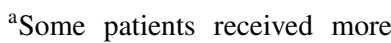
than one agent listed here

\section{Rituximab and COVID-19 antibody development}

By the time of data collection, 29/49 patients (59.2\%) had undergone antibody testing. Fourteen out of the 29 patients tested (48.3\%) had a negative antibody to COVID-19; of note, 4 of the patients who tested negative for antibodies had their antibodies checked less than 21 days from their initial positive SARS-CoV-2 PCR test. In contrast, 15 (51.7\%) of this cohort of 29 patients previously treated with rituximab tested positive for neutralizing antibodies.

The median time between rituximab administration and COVID-19 diagnosis was 58.5 days (4-413 days). Those patients who ultimately developed COVID-19 antibodies

received rituximab a median of 46.0 (4-193) days prior to contracting SARS-CoV-2, while those who did not develop antibodies received rituximab a median of 57.5 (5-413) days prior to infection $(p=0.323)$.

Among the 15 patients who had a documented antibody response, $87 \%(n=13)$ received rituximab within the preceding 6 months. Similarly, 85.7\% $(n=12)$ of those who had documentation of a lack of antibody response received rituximab within 6 months of COVID-19 diagnosis. There was no statistical significance in antibody development between those who were treated with rituximab less than 6 months prior to COVID-19 diagnosis and those who were treated more than 6 months before diagnosis $\left(X^{2}(1\right.$, $n=29)=0.0055, p=0.941)$.

The median time from COVID-19 diagnosis by PCR to antibody testing was 43 days ( 1 to 206 days). For those whose antibodies were positive, the median time between COVID-19 diagnosis and antibody testing was 43.0 days (14-189 days), while the median time between COVID-19 diagnosis and antibody testing for those who tested negative for antibodies was 43.5 days ( $1-206$ days) $(p=0.773)$.

Patients who received other immunosuppressive treatments at the time of their rituximab dosing developed antibodies at similar rates as those who did not receive other concurrent therapies. Eighty percent $(n=12)$ of patients who developed antibodies and $71.4 \%(n=10)$ of those who did not develop antibodies received other concurrent immunosuppressive treatments $\left(X^{2}(1, n=29)=0.2905, p=0.590\right)$.

Seven patients received at least one T-cell-directed therapy in addition to rituximab, including anti-thymocyte globulin (ATG, $n=1)$, cyclosporine $(n=1)$, mycophenolate mofetil (MMF, $n=4)$, and tacrolimus $(n=4)$ (Table 2). Of note, one patient received ATG, MMF, and tacrolimus, and another received both MMF and tacrolimus. Of these patients, only 2 had COVID-19 antibodies checked; the
Table 3 COVID-19 outcomes by time from rituximab to COVID-19 diagnosis

\begin{tabular}{llllll}
\hline \multicolumn{5}{c}{ Time between last rituximab and COVID-19 diagnosis } \\
\cline { 2 - 6 } & $<3$ months & 3-6 months & 6-9 months & 9-12 months & $>12$ months \\
\hline Total $(n=49)$ & $28(57.1 \%)$ & $13(26.5 \%)$ & $5(10.2 \%)$ & $2(4.1 \%)$ & $1(2.0 \%)$ \\
Hospitalization $(n=31)$ & $20(64.5 \%)$ & $6(19.4 \%)$ & $2(6.5 \%)$ & $2(6.5 \%)$ & $1(3.2 \%)$ \\
ICU stay $(n=12)^{\mathrm{a}}$ & $5(41.7 \%)$ & $4(33.3 \%)$ & $2(16.7 \%)$ & $1(8.3 \%)$ & $0(0 \%)$ \\
Death $(n=16)$ & $8(50.0 \%)$ & $5(31.3 \%)$ & $3(18.8 \%)$ & $0(0 \%)$ & $0(0 \%)$ \\
Total patients with anti- & $19(65.5 \%)$ & $6(20.7 \%)$ & $2(6.9 \%)$ & $1(3.4 \%)$ & $1(3.4 \%)$ \\
bodies checked $(n=29)$ & & & & & $1(3.4 \%)$ \\
$+\mathrm{Ab}(n=15)^{\mathrm{b}}$ & $10(34.5 \%)$ & $3(10.3 \%)$ & $0(0 \%)$ & $1(3.4 \%)$ & $1(0 \%)$ \\
\hline $\mathrm{Ab}(n=14)^{\mathrm{b}}$ & $9(31.0 \%)$ & $3(10.3 \%)$ & $2(6.9 \%)$ & $0(0 \%)$ & $0(3)$ \\
\hline
\end{tabular}

ICU intensive care unit, $A b$ COVID-19 antibody

${ }^{a}$ Note that some patients were hospitalized and required ICU level of care, so the columns do not necessarily add up to the totals in the top row

${ }^{b}$ Percentages in these rows are calculated using a denominator of 29 , reflecting the number of patients whose antibodies were checked, not the total number of patients evaluated for this study 
patient who had received ATG, MMF, and tacrolimus did not have detectable antibodies, and an additional patient on only tacrolimus did develop an antibody response.

\section{COVID-19 clinical outcomes}

Over two-thirds (33/49) of the patients in the analysis were hospitalized at the time of their COVID-19 diagnosis. The reason for hospitalization in $87.9 \%$ (29/33) of patients was severe COVID-19 and its complications. Two of the 4 patients hospitalized for other reasons went on to develop severe COVID-19 that ultimately led to their deaths. The remaining two patients hospitalized for reasons other than COVID-19 had only mild infection. For the purpose of this analysis, we considered 31 patients to be hospitalized due to COVID-19- the 29 patients who were admitted due to COVID-19 and the 2 patients who developed severe COVID19 in the course of hospitalizations for other reasons. The 2 patients who developed only mild COVID-19 symptoms during hospitalizations for unrelated medical problems were not included as "hospitalized" patients.

Nineteen $(61.3 \%)$ of those patients hospitalized had received rituximab within 3 months, 6 (19.4\%) received rituximab 3-6 months prior, and 6 (19.4\%) received rituximab more than 6 months before COVID-19 diagnosis. There was no significant difference between hospitalization rates among those who developed COVID-19 antibodies and those who did not develop antibodies $\left(X^{2}(1\right.$, $n=29)=0.9089, p=0.340$ ).

Patients who received rituximab $<3$ months prior to COVID-19 diagnosis were no more likely to be hospitalized than those who were farther out from rituximab treatment $\left(X^{2}(1, n=49)=0.5927, p=0.441\right)$. Those individuals who were treated with rituximab $<3$ months prior to COVID-19 diagnosis were not more likely to have also received other immunosuppressing therapies than those who received rituximab $>3$ months from COVID diagnosis $\left(X^{2}\right.$ $(1, n=49)=0.8725, p=0.350)$.

Nearly a quarter of those in the analysis required a stay in the intensive care unit (ICU) during their COVID-19 hospitalization, all of whom were hospitalized for COVID-19 and not for other reasons. Three quarters (9/12) of those who required ICU care had received rituximab within the prior 6 months, while the remaining 25\% (3/12) received rituximab 6-12 months before infection. There was no significant difference between ICU requirement among those who received rituximab $<3$ months before COVID diagnosis compared to those who received it $>3$ months before COVID diagnosis $\left(X^{2}(1, n=49)=1.5542, p=0.213\right)$. The rates of ICU admissions among patients who developed a COVID-19 antibody response were similar to the rates of ICU stays among those who did not develop antibodies $\left(X^{2}\right.$ $(1, n=29)=0.3326, p=0.564)$.
Thirty-three of the 49 patients evaluated in this study (67.3\%) survived COVID-19 infection; sixteen of those same 49 patients (32.7\%) died prior to data collection (Table 4). Three of the sixteen patients who died were COVID-19 antibody negative. The difference in deaths between the patients who developed antibodies and those who did not was not statistically significant $(p=0.10)$.

\section{Correlation between known risk factors for severe COVID-19 and outcomes}

When comparing outcomes of patients who received rituximab for the treatment of malignancy to those who received it for the treatment of other diseases, the number of patients requiring ICU care for COVID-19 was significantly greater in the non-cancer group. While only $9.5 \%$ of those receiving rituximab for malignant disease required an ICU admission, $35.7 \%$ of those receiving it for non-malignant disease were admitted to the ICU for COVID-19 treatment $\left(X^{2}(1\right.$, $n=49)=4.4512, p=0.035)$. There was no difference in rates of hospitalization among cancer and non-cancer patients $\left(61.9 \%\right.$ v. $71.4 \%$ respectively, $X^{2}(1, n=49)=0.4949$, $p=0.482)$ or death from COVID-19 (23.8\% v. 39.3\%, $X^{2}(1$, $n=49)=1.307, p=0.253$ ).

Among our cohort, age was significantly associated with death from COVID-19, with a relative risk of death in patients age $\geq 60$ years of 3.125 (95\% CI 1.1690 to 8.3536 , $p=0.023$ ). There was no significant relationship between death and other known risk factors for severe COVID-19 infection, such as male gender $\left(X^{2}(1, n=49)=0.5025\right.$, $p=0.48)$, diabetes $\left(X^{2}(1, n=49)=1.7455, p=0.19\right)$, hypertension $\left(X^{2}(1, n=49)=0.1113, p=0.74\right)$, obesity $\left(X^{2}(1\right.$, $n=49)=1.0105, p=0.31)$, or atherosclerotic cardiovascular disease $\left(X^{2}(1, n=49)=3.675, p=0.06\right)$.

Data on absolute lymphocyte count (ALC) at the time of a positive SARS-CoV-2 PCR \pm 3 days was available for 40 of the 49 patients evaluated. Three quarters (30/40) of patients were lymphopenic (defined as an ALC $<0.99 \mathrm{~K} /$ uL obtained in a MSHS clinical laboratory) at the time of COVID-19 diagnosis, with a median ALC of $0.56 \mathrm{~K} / \mathrm{uL}$. Lymphopenia was present in a significant proportion of patients who had received rituximab within 3 months of COVID-19 infection (81.8\%), 3-6 months from infection

Table 4 Deaths by COVID-19 antibody status

\begin{tabular}{lll}
\hline & $\begin{array}{l}\text { Alive at time of } \\
\text { data collection }\end{array}$ & $\begin{array}{l}\text { Deceased at time } \\
\text { of data collection }\end{array}$ \\
\hline Antibody positive, $n(\%)$ & $15(30.6 \%)$ & $0(0 \%)$ \\
Antibody negative, $n(\%)$ & $11(22.5 \%)$ & $3(6.1 \%)$ \\
Antibody status unknown, $n(\%)$ & $7(14.3 \%)$ & $13(26.5 \%)$ \\
Total, $n(\%)$ & $33(67.3 \%)$ & $16(32.7 \%)$ \\
\hline
\end{tabular}


(54.5\%), 6-9 months from infection (75\%), and in all patients who received rituximab $>9$ months from COVID19 diagnosis. There was no significant difference in the rates of lymphopenia among those who received rituximab within 3 months of COVID-19 infection and those who were infected $>3$ months from their last rituximab infusion $\left(X^{2}(1, n=40)=1.2121, p=0.271\right)$.

Lymphopenia was not associated with need for hospitalization $(76.7 \%$ of lymphopenic patients were hospitalized v. $70 \%$ of non-lymphopenic patients, $X^{2}(1$, $n=40)=0.1778, p=0.673)$, ICU admission $(42.9 \%$ in both groups), or death $\left(40 \%\right.$ v. $30 \%, X^{2}(1, n=40)=0.32$, $p=0.572$ ).

There were 21 patients who had ALC data available and had also been tested for COVID-19 antibodies. Baseline lymphopenia at COVID-19 diagnosis was not associated with the development of COVID-19 antibodies: 53.5\% $(8 / 15)$ of patients with lymphopenia went on to develop COVID-19 antibodies, and 50\% (3/6) of patients without baseline lymphopenia developed antibodies $\left(X^{2}(1\right.$, $n=21)=0.0191, p=0.890$ ).

\section{Correlation between titers and outcomes}

COVID-19 antibody titers observed in antibody positive patients are detailed in Table 5. Of the 15 patients who tested positive for antibodies, 8 required hospitalization and had moderate $(1: 320, n=2)$ or high $(\geq 1: 960, n=6)$ antibody titers. Two of those hospitalized required ICU care and developed moderate $(n=1)$ or high $(n=1)$ titers. Seven patients who developed antibodies required no hospitalization or ICU stay and did not die as a result of COVID-19; one of these patients had low antibody titers, 1 had moderate antibody titers and the remaining 5 had

Table 5 COVID-19 antibody titers and outcomes

\begin{tabular}{|c|c|c|c|}
\hline & \multicolumn{3}{|c|}{ COVID-19 antibody titer ${ }^{a}$} \\
\hline & Low $(1: 80)$ & Moderate $(1: 320)$ & $\operatorname{High}(\geq 1: 960)$ \\
\hline $\begin{array}{l}\text { Hospitalization, } \\
n(\%)\end{array}$ & $0(0 \%)$ & $2(13.3 \%)$ & $5(33.3 \%)$ \\
\hline ICU stay, $n(\%)^{\mathrm{b}}$ & $0(0 \%)$ & $1(6.7 \%)$ & $1(6.7 \%)$ \\
\hline Death, $n(\%)$ & $0(0 \%)$ & $0(0 \%)$ & $0(0 \%)$ \\
\hline $\begin{array}{l}\text { No hospitalization, } \\
\text { ICU stay or death, } \\
n(\%)\end{array}$ & $1(6.7 \%)$ & $1(6.7 \%)$ & $6(40.0 \%)$ \\
\hline Total, $n(\%)$ & $1(6.7 \%)$ & $3(20.0 \%)$ & $11(73.3 \%)$ \\
\hline
\end{tabular}

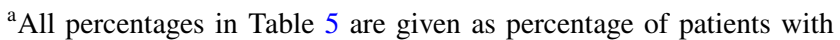
antibodies detected on the COVID-19 antibody assay $(n=15)$

${ }^{\mathrm{b}}$ Individuals who required admission to the ICU were also, by definition, hospitalized high antibody titers. None of the patients who developed antibodies died during the period of the study.

\section{Discussion}

In this series, rituximab therapy in the year prior to COVID19 diagnosis did not significantly impact antibody development or the clinical outcomes evaluated, alone or in combination with other immunosuppressing treatments. The time from COVID-19 diagnosis to antibody testing was not a significant factor in whether patients tested positive for antibodies, and the median time from rituximab administration to COVID-19 diagnosis did not appear to impact antibody development.

Although over two-thirds of patients in this analysis were exposed to additional immunomodulating agents at the time of their rituximab treatment, none of the other chemotherapies or other immunosuppressing treatments have the long duration of effect that rituximab has. However, many of these medications are administered repeatedly over the course of a patient's treatment, and their impact on immune responses to COVID-19 in our cohort is unclear. It is interesting that there was no significant difference in antibody development among those who received concurrent therapies and those who did not, but a further focused analysis of various treatment regimens would be needed to comment further on these agents' effect on COVID-19 immunity and outcomes. While seven patients in our study received T-cell-directed therapies in addition to rituximab, antibody data was lacking for most of these patients, limiting our ability to draw conclusions on the interplay between these two types of immunosuppressants and their effect on the immune response to COVID-19. In the future, it would be interesting to evaluate COVID-19 clinical outcomes and immune responses in patients receiving therapies that affect both B- and T-cell immunity.

Despite the immunosuppressive effects of rituximab, clinical measures of COVID-19 disease severity, such as hospitalization, ICU care, and death, were not significantly affected by the time from rituximab treatment. Similarly, proximity to treatment with rituximab did not appear to affect the development of lymphopenia upon COVID19 diagnosis or the development of a detectable antibody response to COVID-19. Though there is an established association between lymphopenia and death from COVID-19 $[11,12]$, patients with lymphopenia in this study were not more likely to have had fatal outcomes from COVID-19. These findings suggest that the B-cell depleting effects of rituximab may not correlate with more severe COVID-19 infections, but a larger study using matched controls without rituximab exposure would be necessary to fully answer the question of how rituximab influences COVID-19 outcomes. 
It is notable that patients who received rituximab for non-malignant indications had higher rates of ICU stays for COVID-19 compared to those receiving rituximab as a component of cancer therapy $(35.7 \%$ v. $9.5 \%, p=0.035)$ ). The reason for this is unclear, and a larger study comparing these groups would help determine whether this finding is reproduced.

Among the most interesting findings in our analysis was that 11/14 patients who did not develop antibodies in response to COVID-19 recovered from infection, indicating that development of neutralizing antibodies may not be necessary for recovery.

Though B-cell depletion has been implicated as a risk factor for COVID-19 infection-related mortality [13, 14], this series demonstrates that recovery from COVID-19 can occur in the absence of a documented humoral immune response. The absence of neutralizing antibodies in response to SARS-CoV-2 infection does not necessarily imply that these patients did not develop lasting adaptive immunity to the virus; discordant B- and T-cell responses to COVID-19 infection were demonstrated in a UK study of the immune responses in healthcare workers, which showed that the $11 \%$ of subjects who did not develop a humoral response to infection did develop T cells reactive to SARS-CoV-2 antigens [15]. The findings in our study highlight the importance of B-cell-independent mechanisms of viral recovery in patients infected with SARS-CoV-2.

Several case reports have also pointed out the surprising recovery from COVID-19 of patients with acquired or inherited B-cell dysfunction. Two patients in Italy with X-linked hypogammaglobulinemia recovered after prolonged illness from COVID-19 [16]; a patient actively being treated with ocrelizumab—another B-cell depleting agent—recovered fully and without complication from COVID-19 [17], and another patient on rituximab for multiple sclerosis and with a recently documented B-cell count of $0 \%$ had an uneventful recovery [18].

There has been some speculation as to a protective role for rituximab in COVID-19, which may negate the effects B-cell depletion can have on disease recovery. It has been hypothesized that the same anti-spike antibodies that help neutralize the virus may also be responsible in part for the thrombotic, pulmonary, and hyperinflammatory features of severe COVID-19 infection [19, 20].

One important implication for understanding the outcomes and antibody responses of SARS-CoV-2-infected patients who received rituximab is in helping clinicians assess the benefits and timing of COVID-19 vaccination in this population. Several clinical trials have shown that responses to common vaccines are attenuated after antiCD20 therapies, but responses may be more robust to T-celldependent antigens and may be restored with time from treatment, once the tissue compartment of $\mathrm{CD} 20+\mathrm{B}$-cells has been repopulated [21-23]. As a result, the Infectious Disease Society of America recommends delaying most vaccines by 6 months after administering anti-B cell therapy [24]. Optimal timing for COVID-19 vaccination in rituximab-treated patients is unclear, but some have suggested rituximab dose interruption strategies to allow for vaccination and neutralizing antibody development [25]. It is unknown, however, whether these patients could develop T-cell immunity in response to the vaccine, without identifiable antibody responses.

Limitations of this study include its small sample size, the retrospective nature of the analysis, and the fact that data was obtained from a single healthcare system. The spectrum of treatments our patients received in addition to rituximab leads to substantial heterogeneity in the patient population, which affects our ability to isolate rituximab's impact on COVID-19 outcomes in this cohort. The study is also limited by the absence of antibody testing in all individuals included and the inconsistent timing of antibody testing within the cohort.

\section{Conclusions}

This retrospective review demonstrates that clinical outcomes, including hospitalization, ICU admissions, death, and antibody development, were not significantly affected by rituximab treatment in this population, regardless of when treatment was administered. In addition to this, our cohort showed that a large portion of patients recovered from COVID-19 without developing a detectable humoral immune response. What remains to be seen, though, is whether patients who do not develop neutralizing antibodies are at risk for reactivation or reinfection [26], how patients receiving B-cell depleting therapies will respond to SARS-CoV-2 vaccines, and what the role is of concomitant medications and, in particular, T-cell-directed therapies in mediating the immune response to COVID-19. Further investigation of this unique yet diverse patient population of patients who receive rituximab for both malignant and non-malignant conditions is warranted.

Acknowledgements The authors thank Douglas Tremblay, MD for his assistance with the initial project proposal and data collection tools.

Author contribution All authors contributed to the study conception and design. Data collection, analysis, and literature search were performed by Hannah Levavi and Guido Lancman. The first draft of the manuscript was written by Hannah Levavi and all authors commented on previous versions of the manuscript. Hannah Levavi, MD, designed the research study, performed data collection, analyzed the data, and wrote the paper. Guido Lancman, MD, analyzed the data and edited the paper. Janice Gabrilove, MD, conceived of the project, designed the research study, and edited the paper. All authors read and approved the final manuscript. 
Code availability NA

\section{Declarations}

Ethics approval The study was approved by the Program for the Protection of Human Subjects at the Icahn School of Medicine at Mount Sinai.

\section{Consent to participate and publish NA}

Conflict of interest The authors declare no competing interests.

\section{References}

1. Sette A, Crotty S (2021) Adaptive immunity to SARS-CoV-2 and COVID-19. Cell 184(4):861-880. https://doi.org/10.1016/j.cell. 2021.01.007

2. RydyznskiModerbacher C, Ramirez SI, Dan JM, Grifoni A, Hastie KM, Weiskopf D et al (2020) Antigen-specific adaptive immunity to SARS-CoV-2 in acute COVID-19 and associations with age and disease severity. Cell 183(4):996-1012. https://doi.org/10.1016/j. cell.2020.09.038

3. McLaughlin P, Grillo-Lopez AJ, Link BK et al (1998) Rituximab chimeric anti-CD20 monoclonal antibody therapy for relapsed indolent lymphoma: half of patients respond to a four-dose treatment program. J Clin Oncol 16(8):2825-2833. https://doi.org/10.1200/ JCO.1998.16.8.2825

4. Aksoy S, Dizdar O, Hayran M, Harputluoglu H (2009) Infectious complications of rituximab in patients with lymphoma during maintenance therapy: a systematic review and meta-analysis. Leukemia Lymphoma 50(3):357-365. https://doi.org/10.1080/1042819090 2730219

5. Pujadas E, Ibeh N, Hernandez MM, Waluszko A, Sidorenko T, Flores V et al (2020) Comparison of SARS-CoV-2 detection from nasopharyngeal swab samples by the Roche cobas 6800 SARS-CoV-2 test and a laboratory-developed real-time RT-PCR test. J Med Virol 92(9):1695-1698. https://doi.org/10.1002/jmv.25988

6. Cordes AK, Heim A (2020) Rapid random access detection of the novel SARS-coronavirus-2 (SARS-CoV-2, previously 2019-nCoV) using an open access protocol for the Panther Fusion. J Clin Virol 125:104305. https://doi.org/10.1016/j.jcv.2020.104305

7. Mostafa HH, Carroll KC, Hicken R, Berry GJ, Manji R, Smith E et al (2020) Multi-center evaluation of the Cepheid Xpert ${ }^{\circledR}$ Xpress SARS-CoV-2/Flu/RSV Test. J Clin Microbiol 59(3):e02955-e3020. https://doi.org/10.1128/JCM.02955-20

8. Lima A, Healer V, Vendrone E, Silbert S (2020) Validation of a modified CDC assay and performance comparison with the NeuMoDx ${ }^{\mathrm{TM}}$ and DiaSorin ${ }^{\circledR}$ automated assays for rapid detection of SARS-CoV-2 in respiratory specimens. J Clin Virol 133:104688. https://doi.org/10.1016/j.jcv.2020.104688

9. Wajnberg A, Amanat F, Firpo A, Altman DR, Bailey MJ, Mansour $\mathrm{M}$ et al (2020) Robust neutralizing antibodies to SARS-CoV-2 infection persist for months. Science 370(6521):1227-1230. https://doi. org/10.1126/science.abd7728

10. IBM Corp. Released 2020. IBM SPSS Statistics for Macintosh, Version 27.0. Armonk: IBM Corp

11. Ruan Q, Yang K, Wang W, Jiang L, Song J (2020) Clinical predictors of mortality due to COVID-19 based on an analysis of data of 150 patients from Wuhan, China. Intensive Care Med 46(5):846848. https://doi.org/10.1007/s00134-020-05991-x

12. Zhao Q, Meng M, Kumar R, Wu Y, Huang J, Deng Y et al (2020) Lymphopenia is associated with severe coronavirus disease 2019 (COVID-19) infections: a systemic review and meta-analysis. Int J Infect Dis 96:131-135. https://doi.org/10.1016/j.ijid.2020.04.086
13. Lenti MV, Aronico N, Pellegrino I, Boveri E, Giuffrida P, Borrelli de Andreis F et al (2020) Depletion of circulating IgM memory B cells predicts unfavourable outcome in COVID-19. Sci Rep 10(1):20836. https://doi.org/10.1038/s41598-020-77945-8

14. Tepasse PR, Hafezi W, Lutz M, Kühn J, Wilms C, Wiewrodt R et al (2020) Persisting SARS-CoV-2 viraemia after rituximab therapy: two cases with fatal outcome and a review of the literature. $\mathrm{Br} \mathrm{J}$ Haematol 190(2):185-188. https://doi.org/10.1111/bjh.16896

15. Reynolds CJ, Swadling L, Gibbons JM, Pade C, Jensen MP, Diniz MO et al (2020) Discordant neutralizing antibody and T cell responses in asymptomatic and mild SARS-CoV-2 infection. Sci Immunol 5(54):eabf3698. https://doi.org/10.1126/sciimmunol.abf36 98

16. Soresina A, Moratto D, Chiarini M, Paolillo C, Baresi G, Focà E et al (2020) Two X-linked agammaglobulinemia patients develop pneumonia as COVID-19 manifestation but recover. Pediatr Allergy Immunol 31(5):565-569. https://doi.org/10.1111/pai.13263

17. Novi G, Mikulska M, Briano F, Toscanini F, Tazza F, Uccelli A (2020) COVID-19 in a MS patient treated with ocrelizumab: does immunosuppression have a protective role? Mult Scler Relat Disord 42:102-120. https://doi.org/10.1016/j.msard.2020.102120

18 Wurm H, Attfield K, Iversen AK, Gold R, Fugger L, Haghikia A (2020) Recovery from COVID-19 in a B-cell-depleted multiple sclerosis patient. Mult Scler 26(10):1261-1264. https://doi.org/10.1177/ 1352458520943791

19. Liu L, Wei Q, Lin Q, Fang J, Wang H, Kwok H et al (2019) Anti-spike IgG causes severe acute lung injury by skewing macrophage responses during acute SARS-CoV infection. JCI Insight 4(4):e123158. https://doi.org/10.1172/jci.insight.123158

20. Mehta P, Porter JC, Chambers RC, Isenberg DA, Reddy V (2020) B-cell depletion with rituximab in the COVID-19 pandemic: where do we stand? Lancet Rheumatol 2(10):e589-e590. https://doi.org/ 10.1016/s2665-9913(20)30270-8

21. Bedognetti D, Zoppoli G, Massucco C, Zanardi E, Zupo S, Bruzzone A et al (2011) Impaired response to influenza vaccine associated with persistent memory B cell depletion in non-Hodgkin's lymphoma patients treated with rituximab-containing regimens. J Immunol 186(10):6044-6055. https://doi.org/10.4049/jimmunol. 1004095

22. Bar-Or A, Calkwood JC, Chognot C, Evershed J, Fox EJ, Herman A et al (2020) Effect of ocrelizumab on vaccine responses in patients with multiple sclerosis: The VELOCE study. Neurology 95(14):e1999-e2008. https://doi.org/10.1212/WNL.0000000000 010380

23. Bingham CO 3rd, Looney RJ, Deodhar A, Halsey N, Greenwald $\mathrm{M}$, Codding $\mathrm{C}$ et al (2010) Immunization responses in rheumatoid arthritis patients treated with rituximab: results from a controlled clinical trial. Arthritis Rheum 62(1):64-74. https://doi.org/10.1002/ art.25034

24. Rubin LG, Levin MJ, Ljungman P, Davies EG, Avery R, Tomblyn M, Infectious Diseases Society of America et al (2014) 2013 IDSA clinical practice guideline for vaccination of the immunocompromised host. Clin Infect Dis 58(3):309-18. https://doi.org/10.1093/ $\mathrm{cid} / \mathrm{cit} 816$

25. Baker D, Roberts CAK, Pryce G, Kang AS, Marta M, Reyes S et al (2020) COVID-19 vaccine-readiness for anti-CD20-depleting therapy in autoimmune diseases. Clin Exp Immunol 202(2):149-161. https://doi.org/10.1111/cei.13495

26. Lancman G, Mascarenhas J, Bar-Natan M (2020) Severe COVID-19 virus reactivation following treatment for $\mathrm{B}$ cell acute lymphoblastic leukemia. J Hematol Oncol 13(1):131. https://doi.org/10.1186/ s13045-020-00968-1

Publisher's note Springer Nature remains neutral with regard to jurisdictional claims in published maps and institutional affiliations. 